\title{
Outage Probability of Macro-Diversity with Switch-and-Stay Receiver over Gamma-Shadowed Kappa-Mu Multipath Fading Channel
}

\author{
Caslav Stefanovic, Srdjan Jovkovic, Vladeta Milenkovic and Mihajlo Stefanovic
}

\begin{abstract}
In this paper, macro-diversity (MaD) system with macro-diversity switch-and-stay combining (SSC) receiver and two dual input micro-diversity (MiD) maximal-ratio-combing (MRC) structures is considered. Received signal is under the influence of kappa-mu $(k-\mu)$ multipath fading and correlated Gamma long term fading resulting in the system performance degradation. Cumulative density function (CDF) at the output of SSC MaD receiver is obtained and used for the evaluation of the outage probability (OP) of the proposed system. The influence of $k-\mu$ multipath fading severity parameter, line-of-sight (LoS) component, Gamma shadowing severity parameter and shadowing correlation parameter on OP of MaD SSC system are examined and discussed.
\end{abstract}

Index Terms-MaD, MiD, multipath, outage probability, shadowing.

\section{INTRODUCTION}

Macro-diversity $(\mathrm{MaD})$ reception with $\mathrm{MaD}$ receiver and two or more MiD structures can be efficiently used to diminish simultaneously the effects of short-term fading (multipath) and long-term fading (shadowing) on the system performances [1]-[4]. Moreover, $\mathrm{MaD}$ receiver can be applied to reduce shadowing while MiD structures can be applied to reduce multipath on the outage performance.

Although, there are more papers considering first and second order statistics of MaD selection combining (SC) system with maximal-ratio-combining (MRC) at micro structure [5]-[8], there is no paper considering system performances of $\mathrm{MaD}$ switch-and-stay combining (SSC) system with MiD MRC receivers. SSC receiver is often discussed and applied diversity technique due to its relatively low implementation complexity [9]-[10]. MaD SSC system with MaD SSC receiver and two MiD MRC receivers selects MiD MRC receiver to provide service to user for the time being signal envelope at the outputs larger than the given threshold. When signal envelope average power falls below the given threshold, SSC MaD selects the other MRC to give service to user. MRC MiD provides signals addition from

Manuscript received April 8, 2016; revised September 17, 2016

Caslav Stefanovic is with the Faculty of Mathematics and Natural Sciences, University of Pristina, Lole Ribara 29, 38300 Kosovska Mitrovica, Serbia (e-mail: caslav.stefanovic@gmail.com).

Srdjan Jovkovic is with the College of Applied Sciences, Aleksandra Medvedeva, Nis, Serbia.

Vladeta Milenkoovic and Mihajlo Stefanovic are with the Faculty of Electronic Engineering, University of Nis, Aleksandra Medvedeva, 18000, Nis, Serbia (e-mail: vladeta.milenkovic@gmail.com, misa.profesor@gmail.com). both inputs at single base station (BS) aiming to face multipath while SSC MaD selects the input from different BSs aiming to face shadowing.

The Kappa-Mu $(k-\mu)$ distribution describes signal envelope variation in line-of-sight (LoS) multipath fading channels in the environment with two or more clusters. Parameter $k$ is related to availability of LoS propagation between transmitter and receiver of wireless communication channel and can be calculated as the ratio of dominant components power and scattering components power while parameter $\mu$ is related to the number of clusters in the propagation environment. Furthermore, $k-\mu$ is general distribution, which means that Rayleigh, Rice and Nakagami-m distributions for different values of $k-\mu$ parameters can be derived. This distribution accurately fits with experimental data and is often applied in multipath fading environment [11], [12].

On the other hand, Gamma distribution is efficiently used to describe effect of shadowing in wireless communication channel, since it can be advantageous to the log-normal distribution, another well-known distribution often used to describe the effect of shadowing.

In this paper, the sum of one-folded integrals expression of cumulative distribution function (CDF) of MaD SSC receiver output signal envelope in the presence of correlated Gamma shadowed $k-\mu$ multipath fading is obtained and used for derivation of outage probability (OP) of the proposed model. Numerical results are presented graphically and the effect of the system parameters on the proposed $\mathrm{MaD}$ model are examined.

\section{SYSTEM MODEL}

The MaD system with two MiD MRC structures routing signal to the MaD SSC receiver is shown on Fig. 1. The system is under the influence of correlated Gamma shadowed $k-\mu$ multipath fading.

Random variables $x_{i j}, i=1,2 ; j=1,2$; follow $k-\mu$ distribution [13]:

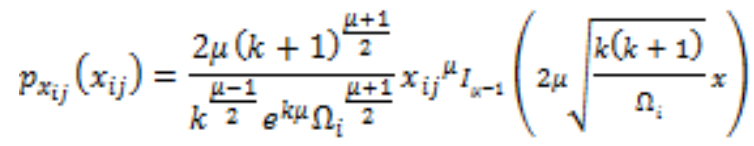

$$
\begin{aligned}
& \cdot e^{-\frac{\mu(k+1)}{n_{i}} x_{i j}{ }^{2}}, i=1,2 ; j=1,2 \text {; }
\end{aligned}
$$

where, $\mu$ is fading severity factor, $k$ is Rice factor, $\Omega_{i}$ is related to the local mean power of $x_{i j}$ and $I_{v}(\cdot)$ is the modified Bessel 
function of the first kind and order $v$ [14].

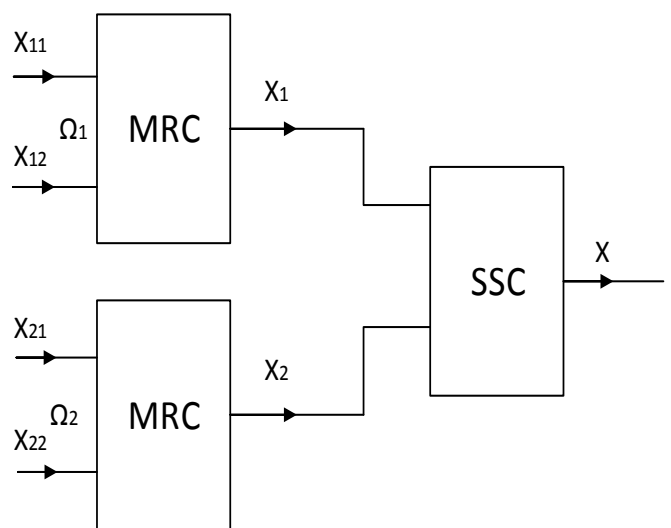

Fig. 1. Block diagram of MaD system with MaD SSC structure and two MiD MRC branches.

The $I_{v}(\cdot)$ can be transformed by utilization [15], so that the probability density function (PDF) of $x_{\mathrm{ij} p} i=1_{x} 2_{x} j=1_{x} 2$; can be expressed as:

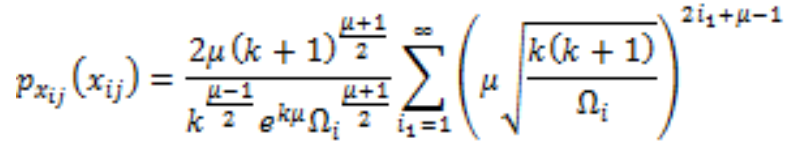

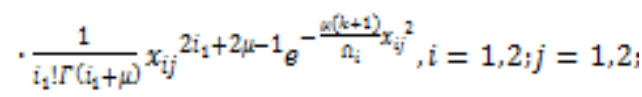

where $\gamma\left(m_{x} x\right)$ is incomplete Gamma function [14] and $\Gamma(\mathrm{s})$ is Gamma function [14]. Signal envelopes at outputs of MiD MRC paths are [16]:

$$
x_{i}^{2}=x_{i j}^{2}+x_{i j}^{2} i=1,2 x
$$

Random variables $x_{i}, i=1,2$; follow $k-\mu$ distribution:

$$
\begin{aligned}
p_{x_{i}}\left(x_{i}\right) & =\frac{4 \mu(k+1)^{\mu+\frac{1}{2}}}{k^{\mu-\frac{1}{2}} e^{2 k \mu}\left(2 \Omega_{\mathrm{i}}\right)^{\mu+\frac{1}{2}}} \sum_{i_{1}=1}^{m}\left(2 \mu \sqrt{\frac{k(k+1)}{2 \Omega_{\mathrm{i}}}}\right)^{2 i_{1}+2 \mu-1} \\
& =\frac{1}{i_{1} ! N\left(i_{1}+2 \mu\right)} x_{i j}{ }^{2 i_{1}+4 \mu-1} e^{-\frac{\mu(k+1)}{\Omega_{i}} x_{i j}{ }^{2}}, i=1,2
\end{aligned}
$$

Further, cumulative distribution function (CDF) of $x_{i}, i=1,2 x$ is [16]:

$$
\begin{gathered}
F_{x_{1}}\left(x_{\mathrm{i}}\right)=\int_{0}^{x_{\mathrm{i}}} p_{x_{\mathrm{i}}}(t) d t \\
=\frac{4 \mu(k+1)^{\mu+\frac{1}{2}}}{k^{\mu-\frac{1}{2}} e^{2 k \mu}\left(2 \Omega_{\mathrm{i}}\right)^{\mu+\frac{1}{2}}} \sum_{i_{1}=1}^{m}\left(2 \mu \sqrt{\frac{k(k+1)}{2 \Omega_{\mathrm{i}}}}\right)^{2 i_{1}+2 \mu-1} \\
\frac{1}{i_{1} ! \Gamma\left(i_{1}+2 \mu\right)} \frac{1}{2}\left(\frac{\Omega_{\mathrm{i}}}{\mu(k+1)}\right)^{i_{1}+2 \mu} \gamma\left(i_{1}+2 \mu, \frac{\mu(k+1)}{n_{\mathrm{i}}} x_{\mathrm{i}}^{2}\right) .
\end{gathered}
$$

Signal envelopes average power at inputs of MiD MRC structures, $\Omega_{1}$ and $\Omega_{2}$ follow joint correlated Gamma distribution [17], [18]:

$$
\begin{gathered}
p_{\Omega_{1} \Omega_{2}}\left(\Omega_{1} \Omega_{2}\right)=\frac{\left(\Omega_{1} \Omega_{2}\right)^{\frac{c-1}{2}}}{\Gamma(c)\left(1-\rho^{2}\right) \rho^{c-1} \Omega_{0}^{c+1}} \\
=\frac{e^{-\frac{n_{1}+n_{2}}{M_{0}\left(1-p^{2}\right)}} I_{c-1}\left(\frac{2 \rho}{n_{0}\left(1-\rho^{2}\right)}\left(\Omega_{1} \Omega_{2}\right)^{\frac{1}{2}}\right)}{\Gamma(c)\left(1-\rho^{2}\right) \rho^{c-1} \Omega_{0}{ }^{c+1}} \sum_{i=0}^{\infty}\left(\frac{\rho}{\Omega_{0}\left(1-\rho^{2}\right)}\right)^{2 i+c-1} \\
=\frac{1}{i ! r(i+c)} \Omega_{1}^{i+c-1} \Omega_{2}^{i+c-1} e^{-\frac{n_{1}+n_{2}}{n_{0}\left(1-p^{2}\right)}}
\end{gathered}
$$

where $p$ is correlation coefficient of the shadowing process, $c$ is shadowing parameter and $\Omega_{0}$ is mean value of $\Omega_{1}$ and $\Omega_{2}$.

CDF of MaD SSC output signal envelope is equal to the CDF of the first MiD MRC output signal envelope when the first MiD MRC provides signal to mobile user and the total power at its inputs is higher than predetermined threshold or when the second MiD MRC receiver provides signal to the mobile user and the total power at its inputs is lower than the threshold. Contrary, CDF of MaD SSC output signal envelope is equal to $\mathrm{CDF}$ of the second MiD MRC output signal envelope when the second MiD MRC provides the signal to mobile user and the total power at its inputs is higher than the predetermined threshold or when the first MiD MRC provides the mobile user and the total power at its input is lower than the predetermined threshold.

Accordingly, CDF of MaD SSC receiver output signal envelope is:

$$
F_{x}(x)=\frac{1}{2} \int_{\Omega_{T}}^{m} d \Omega_{1} \int_{0}^{m \infty} F_{x_{1}}\left(X \mid \Omega_{1}\right) p_{\Omega_{1} \Omega_{2}}\left(\Omega_{1} \Omega_{2}\right) d \Omega_{2}
$$

$$
\begin{aligned}
& +\frac{1}{2} \int_{0}^{\Omega_{T}} d \Omega_{1} \int_{0}^{\infty} F_{x_{2}}\left(X \mid \Omega_{2}\right) p_{\Omega_{1} \Omega_{2}}\left(\Omega_{1} \Omega_{2}\right) d \Omega_{2} \\
& +\frac{1}{2} \int_{\Omega_{T}}^{\infty} d \Omega_{2} \int_{0}^{\infty} F_{x_{2}}\left(X \mid \Omega_{2}\right) p_{\Omega_{1} \Omega_{2}}\left(\Omega_{1} \Omega_{2}\right) d \Omega_{1} \\
& +\frac{1}{2} \int_{0}^{\Omega_{T}} d \Omega_{2} \int_{0}^{\infty} F_{x_{1}}\left(X \mid \Omega_{1}\right) p_{\Omega_{1} \Omega_{2}}\left(\Omega_{1} \Omega_{2}\right) d \Omega_{1} \\
& =\int_{\Omega_{T}}^{\infty} d \Omega_{1} \int_{0}^{\infty \infty} d \Omega_{2} F_{x_{1}}\left(X \mid \Omega_{1}\right) p_{\Omega_{1} \Omega_{2}}\left(\Omega_{1} \Omega_{2}\right) \\
& +\int_{0}^{\Omega_{T}} d \Omega_{1} \int_{0}^{\infty} d \Omega_{2} F_{x_{2}}\left(X \mid \Omega_{2}\right) p_{\Omega_{1} \Omega_{2}}\left(\Omega_{1} \Omega_{2}\right) \\
& =S_{1}+S_{2} .
\end{aligned}
$$

where $F_{X] n_{1}}$ is CDF at the first input of SSC and $F_{X] \Omega_{2}}$ is CDF at the second input of SSC.

Integral of the first sum $S_{1}$ is [14]: 


$$
\begin{aligned}
& S_{1}=\int_{\Omega_{T}}^{m} d \Omega_{1} \int_{0}^{\infty} F_{x_{1}}\left(X \mid \Omega_{1}\right) p_{\Omega_{1} \Omega_{2}}\left(\Omega_{1} \Omega_{2}\right) d \Omega_{2}= \\
& =\frac{4 \mu(k+1)^{\mu+\frac{1}{2}}}{k^{\mu-\frac{1}{2}} e^{2 k \mu} 2^{\mu+\frac{1}{2}}} \sum_{i_{1}=1}\left(2 \mu \sqrt{\frac{k(k+1)}{2}}\right)^{2 i_{1}+2 \mu-1} \\
& \times \frac{1}{i_{1} ! \Gamma\left(i_{1}+2 \mu\right)} \frac{1}{2}\left(\frac{1}{\mu(k+1)}\right)^{i_{1}+2 \mu} \frac{1}{\Gamma(c)\left(1-\rho^{2}\right) \rho^{c-1} \Omega_{0}{ }^{c+1}} \\
& =\sum_{i_{2}=0}^{\infty}\left(\frac{\rho}{\Omega_{0}\left(1-\rho^{2}\right)}\right)^{2 i_{2}+c-1} \frac{1}{i_{2} ! \Gamma\left(i_{2}+c\right)} \\
& \cdot\left(\Omega_{0}\left(1-\rho^{2}\right)\right)^{i_{2}+c} \Gamma\left(i_{2}+c\right) \\
& \cdot \int_{\Omega_{Y}}^{\infty} \Omega_{1}^{i_{1}+\alpha-1} \gamma\left(i_{1}+2 \mu \frac{\mu(k+1)}{\Omega_{1}} x^{2}\right) e^{-\frac{a_{1}}{a_{Q}\left(1-p p^{2}\right)}} d \Omega_{1}
\end{aligned}
$$

Integral of the second sum $S_{2}$ is [14]:

$$
\begin{aligned}
& S_{2}=\int_{0}^{\Omega_{T}} d \Omega_{1} \int_{0}^{m \infty} F_{X_{2}}\left(X \mid \Omega_{1}\right) p_{\Omega_{1} \Omega_{2}}\left(\Omega_{1} \Omega_{2}\right) d \Omega_{2} \\
& =\frac{4 \mu(k+1)^{\mu+\frac{1}{2}}}{k^{\mu-\frac{1}{2}} e^{2 k \mu} 2^{\mu+\frac{1}{2}}} \sum_{i_{1}=1}\left(2 \mu \sqrt{\frac{k(k+1)}{2}}\right)^{2 i_{1}+2 \mu-1} \\
& \times \frac{1}{i_{1} ! \Gamma\left(i_{1}+2 \mu\right)} \frac{1}{2}\left(\frac{1}{\mu(k+1)}\right)^{i_{1}+2 \mu} \frac{1}{\Gamma(c)\left(1-\rho^{2}\right) \rho^{\complement-1} \Omega_{0}{ }^{c+1}} \\
& =\sum_{i_{2}=0}^{\infty}\left(\frac{\rho}{\Omega_{0}\left(1-\rho^{2}\right)}\right)^{2 i_{2}+c-1} \frac{1}{i_{2} ! \Gamma\left(i_{2}+c\right)} \\
& =\left(\Omega_{0}\left(1-\rho^{2}\right)\right)^{i_{2}+c}{ }_{\gamma}\left(i_{2}+c_{s} \frac{\Omega_{T}}{\Omega_{0}\left(1-\rho^{2}\right)}\right) \\
& \cdot \int_{0}^{\infty} \Omega_{2}^{i_{2}+c-1} \gamma\left(i_{1}+2 \mu \mu_{s}^{\mu(k+1)} \frac{n_{2}}{\Omega_{2}} x^{2} e^{-\frac{\Omega_{2}}{a_{2}\left(1-x^{2}\right)}} d \Omega_{2} .\right.
\end{aligned}
$$

\section{NUMERICAL RESULTS}

Outage probability (OP), defined as the probability that the output signal envelope of MaD SSC receiver drops below given outage threshold $\Omega_{T}$, is given as [16]:

$$
P_{\text {out }}=\int_{0}^{\Omega_{T}} p_{X}(t) d t=F_{X}\left(\Omega_{T}\right)
$$

After substituting (7) in (10), OP is obtained in the form of the sum of one-folded integrals which are solved using software package mathematica and graphically presented for various system parameters on Fig. 2-Fig. 4.

OP of MaD SSC system for various values of Rice factor $k$, fading severity parameters $\mu$ and shadowing severity parameter $c$ and for constant values of correlation parameter $\rho$, average power $\Omega_{0}$ and threshold value $\Omega_{T}$ is presented on Fig. 2 . It can been seen that by increasing parameter $c$, the performances improves, since OP decreases. Moreover, the system performance improvement is also evident by increasing parameters $\mu$ and $\mathrm{k}$. It's obvious that parameter $c$ has greater impact on OP then parameter $\mu$ and $k$. Further, the Rice factor has greater impact then the severity of multipath fading.

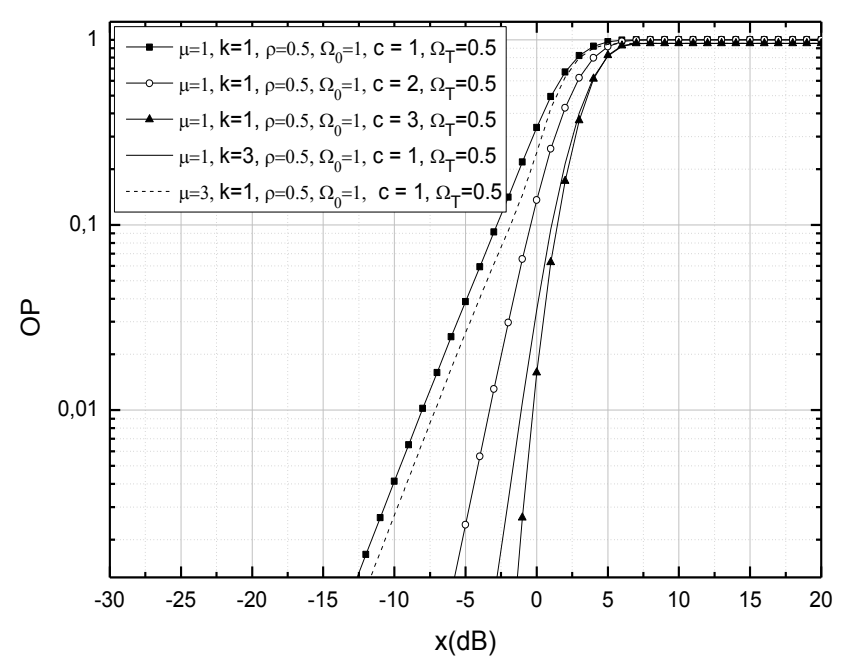

Fig. 2. OP of MaD SSC receiver for different values of parameter $\rho$ and constant values of $\mu, k, c, \Omega_{\mathrm{s}}$ and $\Omega_{0}$.

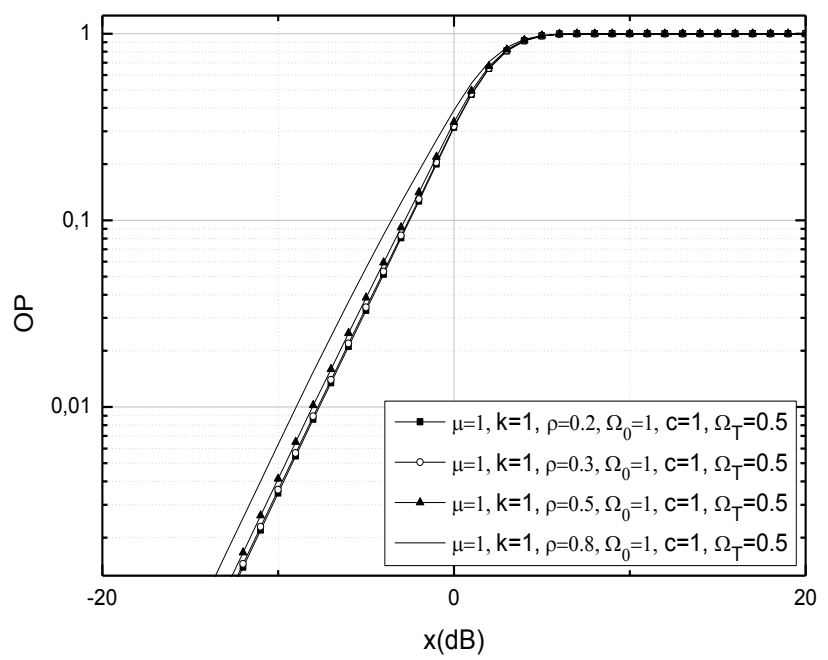

Fig. 3. OP of MaD SSC receiver for different values of parameter $\rho$ and constant values of $\mu, \mathrm{k}, \mathrm{c}, \Omega_{\mathrm{r}}$ and $\Omega_{0}$.

Fig. 3 shows OP of MaD SSC system for constant values $k$, $\mu, c, \Omega_{T}$ and $\Omega_{0}$ and different values of $\rho$. Since, there is no correlation for $\rho=0$, the influence of correlation on the $\mathrm{MaD}$ SSC system performances increases for higher values of $\rho$, resulting in system performance degradation, as expected. Since, OP decreases by decreasing parameter $\rho$ influence of correlation on the performances cannot be neglected for higher values of $\rho$, while it is without significant importance for lower values of $\rho$. 
OP of MaD SSC system for constant values $k, \mu, c, \rho$ and $\Omega_{0}$ and different values of $\Omega_{T}$ is shown on Fig. 4. OP decreases by decreasing parameter $\Omega_{T}$, which means that for lower values of $\Omega_{T}$, MaD SSC receiver is more stable since it has less switches between MiD MRC paths.

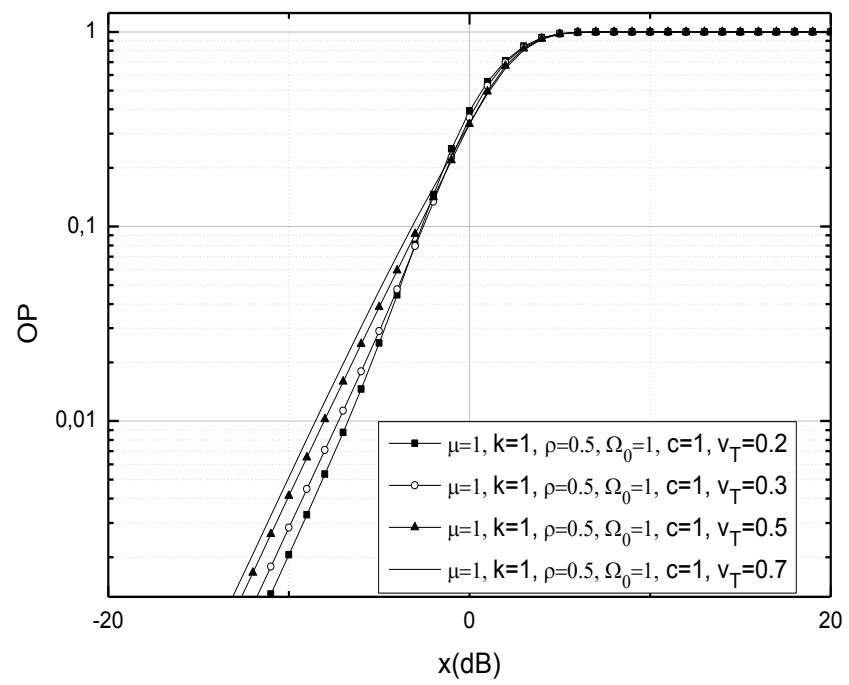

Fig. 4. OP of MaD SSC receiver for different values of parameter $\Omega_{T}$ and constant values of $\mu, \mathrm{k}, \mathrm{c}, \rho$ and $\Omega_{0}$.

\section{CONCLUSION}

In this paper, $\mathrm{MaD}$ technique with $\mathrm{MaD}$ switch and stay combining reception and two MiD selection combiners in correlated Gamma shadowed Kappa-Mu multipath fading channel is considered. MaD system with MaD SSC receiver is proposed, since it has lower complexity implementation then other diversity techniques. OP of SSC MaD system in the form of the sum of one-folded integral is obtained and graphically presented in relation to different system model parameters. As expected, the proposed model is theoretically more efficient by modeling with higher values of parameter $\mathrm{c}$, $k$ and $\mu$. Moreover, it can be concluded that parameter $c$ has greater impact on the system performances than parameters $\mu$ and $k$. Moreover, influence of correlation parameter $\rho$ and threshold of SSC receiver $\Omega_{T}$ on the MaD proposed model is also presented and investigated. In general the best possible outcome in theory is achievable by decreasing parameters $\rho$ and $\Omega_{T}$ and by increasing $\mathrm{c}, k$ and $\mu$.

\section{REFERENCES}

[1] D. A. Basnayaka, P. J. Smith, and P. A. Martin, "The effect of macro diversity on the performance of maximal ratio combining in flat Rayleigh fading," IEEE Transactions on Communications, vol. 61, no. 4, pp. 1384-1392, 2013.

[2] B. Zhang, Z. Zhong, B. Ai, and R. He, "Impact of shadowing correlation on microdiversity and marcodiversity of cellular system in high-speed railway environments," in Proc. Progress In Electromagnetics Research Symposium, Guangzhou, China, Aug. 25-28, 2014, pp. 2159-2163.

[3] D. Djosic, D. Stefanovic, and C. Stefanovic, "Level crossing rate of macro-diversity system with two micro-diversity $\mathrm{SC}$ receivers over correlated gamma shadowed $\alpha-\mu$ multipath fading channels," IETE Journal of Research, pp. 1-6, 2015.

[4] Č. Stefanović, "Macrodiversity system with macrodiversity SSC receiver and two microdiversity receivers in the presence of composite fading environment," in Proc. $23^{\text {rd }}$ Telecommunications Forum -
TELFOR 2015, Proceedings of papers, Belgrade, Serbia, 24-26, 2015, pp. 321-324.

[5] S. Mukherjee and D. Avidor, "Effect of microdiversity and correlated macrodiversity on outages in a cellular system," IEEE Trans. on Wireless Technol., vol. 2, no. 1, pp. 50-59, 2003.

[6] P. M. Shankar, "Macrodiversity and microdiversity in correlated shadowed fading channels," IEEE Trans. on Vehicular Technol., vol. 2, no. 58, pp. 727-732, 2009.

[7] N. Sekulović and M. Stefanović, "Performance analysis of system with micro-and macrodiversity reception in correlated gamma shadowed Rician fading channels," Wireless Personal Communications, vol. 65, no. 1, pp. 143-156, 2012.

[8] S. Panić, D. Stefanovic, I. Petrović, M. Stefanović, J. Anastasov, and D. Krstic, "Second order statistics of selection macro-diversity system operating over Gamma shadowed k- $\mu$ fading channels," EURASIP Journal on Wireless Communications and Networking, vol. 2011, Oct. 2011.

[9] N. C. Beaulieu, "Switching rates of dual selection diversity and dual switch-and stay diversity," IEEE Transaction On Communications, vol. 56, no. 9, pp. 1409-1413, 2008.

[10] J. Zhao, P. Fan, N. C. Beaulieu, and X. Lei, "Switching rates of selection diversity and switch-and stay diversity on mixed high-speed train channels," in Proc. IEEE International Workshop on High Mobility Wireless Communications, Xi'an, China, 21-23 Oct. 2015, pp. 51-55.

[11] M. D. Yacoub, "The k- $\mu$ distribution and the $\eta-\mu$ distribution," IEEE Antennas and Propagation Magazine, vol. 49, no. 1, pp. 68-81, 2007.

[12] X. Wang and N. Beaulieu, "Switching rates of two-branch selection diversity in $\kappa-\mu$ and $\alpha-\mu$ distributed fadings," IEEE Trans Wireless Commun., vol. 8, no. 4, pp. 1667-1671, 2009.

[13] S. Panic, M. Stefanovic, J. Anastasov, and P. Spalevic, Fading and Interference Mitigation in Wireless Communications, CRC Press: New York, 2013.

[14] I. S. Gradshteyn and I. M. Ryzhik, Table of Integrals, Series, and Products, $6^{\text {th }}$ ed., New York: Academic, 2000.

[15] The Wolfarm Functions Site, 2008. [Online]. Available: http:/functions.wolfarm.com

[16] G. L. Stüber, Principles of Mobile Communications, Kluwer Academic Publishers: Massachusetts, USA, 1996.

[17] E. Xekalaki, J. Panaretos, and S. Psarakis, "A predictive model evaluation and selection approach - the correlated gamma ratio distribution," in Stochastic Musings: Perspectives from the Pioneers of the Late 20th Century, J. Panaretos, Ed. Psychology Press, USA, pp. 188-202, 2003.

[18] S. Yue, T. B. M. J. Ouarda, and B. Bobee, "A review ofbivariate gamma distributions for hydrological application," J. Hydrol., vol. 246, pp. 1-18, 2001.

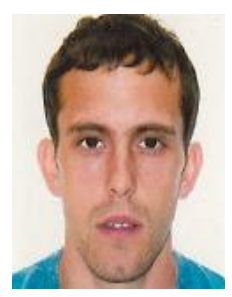

Caslav Stefanovic was born on Jan. 28, 1982 in Nis Serbia. Caslav is currently a Ph.D. candidate at the Faculty of Electronic Engineering, University of Nis, Serbia.

At the moment he is working as assistant at the faculty of Natural Sciences and Mathematics, University of Pristina, Department of Informatics a Kosovska Mitrovica, Serbia. He was a visiting student to the Department of Physics at University of Hamburg in 2009 and Department of Electrotechnics at Technical University in Berlin from 2010 to 2011. His major research interests include wireless and mobile communications and computer networks.

Caslav Stefanovic has more than 25 publications in international and national journals, conferences and seminars.

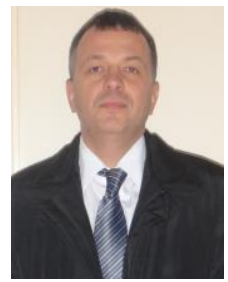

Srdjan Jovkovic was born in Nis, Serbia in 1971.

He received his M.S. and Ph.D. degrees in telecommunication from the Faculty of Electronic Engineering, University of Nis, Serbia in 2006 and 2011, respectively.

He is currently a professor at the Collage of Applied technical Sciencies in Nis, Serbia. His major research interests include wireless and optical communications, mobile and computer networks. 


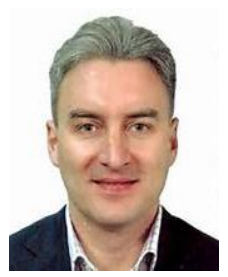

Vladeta Milenkovic graduated in 1992 at the Faculty of Electronic Engineering University of Nis. He received his M.S. degree in Metrology and Measurment Technique in 1999 at the same university. At the moment he is working as assistant at the faculty of Faculty of Electronic Engineering University of Nis.

$\mathrm{He}$ was engaged on several national and practical realizations. international projects including theoretical and

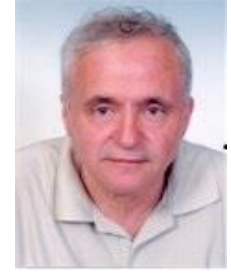

Mihajlo Stefanovic was born in 1947 in Nis, Serbia. $\mathrm{He}$ received his M.S. and Ph.D. degrees in telecommunication from Faculty of Electronic Engineering, University of Nis, Serbia in 1976 and 1979 respectively. He is elected full Professor 1990 at the same university. His major research interests include wireless and optical communications, mobile and computer networks. His research interests in the field of mobile and multichannel communications include statistical characterization and modeling of fading channels, performance analysis of diversity combining techniques, and outage analysis of multiuser wireless systems subjected to interference.

Prof. Stefanovic has more than 320 paper published in significant international and national journals and conferences with more than 1000 citations. 